\title{
Realitätsverschiebungen: Politische und verfassungsrechtliche Dimensionen von Augmented und Virtual Reality
}

\section{Juristische als virtuelle Lebenswelt}

Was ist gemeint, wenn von virtuellen Lebenswelten die Rede ist? Aus juristischer Perspektive steht bei der Beantwortung dieser Frage einiges auf dem Spiel. Denn selbst wenn man nicht gleich die gesamte Rechtsordnung als eine virtuelle Lebenswelt begreifen will, haben doch zentrale Rechtsfiguren und Rechtsinstitute einen virtuellen Charakter: die natürliche Person zum Beispiel also das normative Verständnis von uns selbst; und erst recht die juristische Person - also die normative Verselbständigung von Personenmehrheiten oder Vermögensmassen zu einem juristisch handlungsfähigen Subjekt. Mit dem Begriff der „Virtualität“ wird aber auch die demokratische Repräsentation des Volkes im Deutschen Bundestag beschrieben oder die Rechtstatsache, dass Gerichtsurteile im Namen des Volkes ergehen. Juristinnen und Juristen können mit dieser virtuellen Form grundlegender Rechtskategorien wie ,Person', ,Demokratie‘ und ,Repräsentation' sehr flexibel und phantasievoll umgehen, weil die Rechtswissenschaft und die Rechtspraxis normativ und damit konstruktiv sind. „Im Bereich des Rechts“ - so Bruno Latour (2010, 339) - „besaß man immer die Höflichkeit, den eigenen Relativismus und Konstruktivismus anzuerkennen, ohne eine Staatsaffäre daraus zu machen.“

In gewisser Weise stimmt also durchaus, was viele schon immer vermutet haben: Juristinnen und Juristen reisen wissenschaftlich und praktisch mit sehr leichtem - fast könnte man sagen - virtuellem Gepäck. Sie verfügen über kein real-substanzielles, sondern ein konstruktiv-normatives Verständnis von rechtlicher Realität - also der virtuellen Sphäre, in der sich empirische und normative Welt begegnen. Deshalb können sie Fiktionen, Metaphern und Symbole - wie beispielsweise die natürliche bzw. juristische Person oder demokratische Repräsentation - verwenden, um soziale und politische, ökonomische und ökologische Fragen zu beantworten (Münkler 2016). Allerdings entfaltet sich dieser normative Konstruktivismus keineswegs willkürlich: Zwar kann er sich nicht auf reale Substrate berufen, um juristische Rechtsfiguren und Rechtsinstitute zu begründen: Es darf nicht unmittelbar von einem Sein auf ein Sollen geschlossen werden. Dies wäre ein naturalistischer Fehlschluss, selbst wenn - wie wir sogleich sehen 
werden - die „normative Kraft des Faktischen“ (Jellinek 1914, 338) gerade in der rechtlichen Reflexion der Kommunikationstechnik- und Medienentwicklung eine ganz zentrale Rolle spielt. Doch ungeachtet dessen folgt der rechtliche Konstruktivismus normativen Regeln, die sich insbesondere in der Verfassung, also im Grundgesetz finden. So folgt - wiederum beispielhaft - die normative Konstruktion und rechtliche Ausgestaltung der natürlichen Person - also unseres rechtlichen Status als Menschen - dem Prinzip der Menschenwürde (Artikel 1 Absatz 1 Grundgesetz) und dem Persönlichkeitsrecht (Artikel 2 Absatz 1 in Verbindung mit Artikel 1 Absatz 1 Grundgesetz); sehr verkürzt: Wir müssen als Menschen das Recht haben, uns selbst zu bestimmen und unsere Persönlichkeit zu entwickeln. Ebenso folgt die normative Entfaltung des Volkes als demokratisches Subjekt (Artikel 20 Absatz 2 Satz 1 Grundgesetz) den verfassungsrechtlichen Verfahrensregelungen der demokratischen Wahl der Abgeordneten des Bundestags. Diese repräsentieren aufgrund ihres freien Mandats das ganze Volk (Artikel 38 Grundgesetz) - wiederum sehr verkürzt: Aufgrund ihrer Menschenwürde und ihres Persönlichkeitsrechts können die Bürger`innen sich individuell selbst bestimmen und verfügen deshalb auch über das Recht, sich kollektiv demokratisch selbst zu bestimmen.

Doch mit dieser Offenlegung des konstruktivistischen Verständnisses des Rechts sind die Möglichkeiten, aus juristischer Perspektive die virtuelle Lebenswelt der Rechtsordnung zu beschreiben und zu verstehen, noch keineswegs ausgeschöpft. Denn die virtuelle Realität praktisch aller soeben genannten juristischen Konzepte - also der natürlichen und juristischen Person oder der demokratischen Repräsentation des Volkes im Parlament - wird durch Medien konstituiert oder hängt jedenfalls von Medien ab (Benjamin 2017 [1936], 44 Fußnote 20). Ihre konkrete rechtliche Form und Gestalt beruht auf einem ganz konkreten Medieneinsatz, einer bestimmten Medienverfassung (Vismann 2011, 2012). Aus diesem Grund lässt sich in einer kulturtechnisch informierten juristischen Perspektive das Verhältnis von Recht und Medien noch näher bestimmen: Die virtuelle Realität von Rechtsfiguren und Rechtsinstituten verschiebt sich mit der Medienentwicklung. So verändert sich auch das, was individuell Persönlichkeit und kollektiv Demokratie ist, sein kann oder sein soll, mit den Medien, die einer Gesellschaft und damit deren Rechtsordnung zur Verfügung stehen: Vom Gesprochenen, Gemeißelten und Geschriebenen über das Gedruckte und Gesendete zum Gemailten, Gestreamten und Getwitterten. Diese Feststellung ist an sich banal, doch nicht das, was juristisch aus ihr folgt: Die Rechtsordnung muss sich bei jeder neuen Medienentwicklung entscheiden, ob sie - ganz im Sinn des funktionalen Rechtsverständnisses Niklas Luhmanns (1987, 31-80) - an der überkommenen Medienverfassung ihrer Rechtsfiguren und Rechtsinstitute festhält und diese folglich entgegen einer neuen Medien- 
entwicklung kontrafaktisch stabilisiert. Alternativ kann die Rechtsordnung aber auch medial lernen, also neue Medien in ihr konstruktivistisches Verständnis von Rechtsfiguren und Rechtsinstituten einbeziehen und damit deren normative Form und rechtliche Gestalt verändern. Auf diese Weise führen mediale Entwicklungen über die normativen Konstruktionen zu rechtlichen Realitätsverschiebungen - oder eben nicht. Das wohl bekannteste Beispiel hierfür bildet $\S 169$ Gerichtsverfassungsgesetz: Die gerichtliche Verhandlung und die Verkündung der Urteile und Beschlüsse sind öffentlich. Ton- und Fernsehaufnahmen sind aber grundsätzlich unzulässig, wobei allerdings inzwischen Ausnahmen vorgesehen sind - etwa für die Entscheidungsverkündung oberster Gerichtshöfe oder zu wissenschaftlichen und historischen Zwecken (vgl. auch $\S 17 a$ Bundesverfassungsgerichtsgesetz). Das heißt: Die Rechtsordnung hält nach wie vor für die Gerichtsverhandlung und das Urteil im Namen des Volkes medial am Gesprochenem und Geschriebenen fest. Rundfunk- und Fernsehaufnahmen werden grundsätzlich rechtlich ausgeschlossen bzw. nur unter engen Voraussetzungen ausnahmsweise zugelassen. Wie folgenreich diese Entscheidung über die mediale Verfassung von Rechtsinstituten für die Realität des Gerichtsprozesses ist, kann man sich unmittelbar selbst verdeutlichen, indem man sich den ersten Prozess von O. J. Simpson einmal mit und einmal ohne Fernsehübertragungen der Gerichtsverhandlungen vorstellt.

Im Folgenden soll es um die normativen Realitätsverschiebungen gehen, die Augmented und Virtual Reality verursachen (können). Wie kann und soll unsere Rechtsordnung auf Augmented und Virtual Reality reagieren: kontrafaktisch ablehnend oder positiv lernend? Wie verändern sich Rechtsfiguren und Rechtsinstitute, wenn wir diese medialen Realitätsverschiebungen rechtlich zulassen? Und wie sollen und können wir diese virtuellen Realitätsverschiebungen rechtlich gestalten? Bei der Beantwortung dieser Fragen stehen wir vor dem Problem, dass sowohl Augmented als auch Virtual Reality Kommunikationstechniken sind, die sich gegenwärtig noch auf der Suche nach ihren Anwendungsfeldern befinden (Lanier 2018, 286). Aktuell sind sie noch Teil des medialen Kommunikationsüberschusses, den die digitale Revolution erzeugt hat und mit dem die „nächste Gesellschaft“ erst noch umgehen lernen muss (Baecker 2007, 8-9; 2018, 10-11). Im Hinblick auf Augmented Reality ist Google Glass dafür ein gutes Beispiel: Brillen soll(t)en mittels eines integrierten Minicomputers Personen die Möglichkeit eröffnen, soziale Interaktion mit Daten und Informationen zu unterlegen und verdeckt Bild- und Wortaufnahmen zu machen, die wiederum von dem Brillenhersteller gespeichert werden. Doch Google Glass hat sich nicht durchgesetzt, weil es nicht als eine soziale Technik wahrgenommen wurde (Lanier 2018, 262-263; Bailenson 2018, 10; Volland 2018, 159; Metz und Seeßlen 2018, 110-112). In gewisser Weise spiegelt sich dies 
auch in der juristischen Diskussion, in der die Möglichkeit einer brillengestützten Surveillance als Verstoß gegen das Persönlichkeitsrecht der betroffenen Personen eingeordnet wird - konkreter: als Verstoß gegen das Recht am eigenen Bild und am eigenen Wort, gegen das Recht auf informationelle Selbstbestimmung sowie den Datenschutz (Solmeckeund Kocatepe 2014). Doch zugleich ist auch klar, dass sich ganz parallele rechtliche Einwände bei der Einführung von Mobiltelefonen mit Kamera- und Sprachaufzeichnungsfunktion gestellt haben. Allerdings sind die Medienentwicklung und Mediennutzung in diesem Fall aber schlicht faktisch über die genannten rechtlichen Einwände hinweggegangen (Solmeckeund Kocatepe 2014, 26-27). Dies hat zu einem medialen Strukturwandel der Öffentlichkeit geführt, der die rechtliche Realität für die Gewährleistung unseres Persönlichkeitsrechts verschoben hat. Die normative Kraft des Faktischen - im konkreten Fall der Kommunikationstechnik- und Medienentwicklung - darf also keineswegs unterschätzt werden, wenn rechtliche Realitätsverschiebungen juristisch zu reflektieren sind. Der mediale Übergang von „Überwachen und Strafen“ (Foucault 1976) $\mathrm{zu}$ „Unterwachen und Schlafen“ (Andreas et al. 2018; Rieger 2019, 99-119) vollzieht sich hier nicht durch eine explizite und punktuelle Entscheidung der Rechtsordnung, sondern subkutan und fließend.

Wenn aber Augmented und Virtual Reality erst noch ihre Anwendungsfelder suchen, können diese auch politisch sein. Welche Realitätsverschiebungen in der Rechtsordnung sind hier zu erwarten? Wo wird vor allem das Verfassungsrecht lernen und sich Augmented und Virtual Reality gestaltend annehmen? Und wo wird das Grundgesetz - wahrscheinlich - kontrafaktisch an der überkommenen Medienordnung und damit auch an tradierten rechtlichen Konstruktionen festhalten? Es versteht sich von selbst, dass die Beantwortung dieser Fragen etwas Spekulatives hat: Wer weiß schon wirklich, in welche Richtung sich Augmented und Virtual Reality im Spannungsfeld zwischen virtuellem Realismus und virtuellem Idealismus in unserer disruptiven Medienwelt entwickeln (Lanier 2018, 238)? Doch es gibt immerhin Anhaltspunkte, um erste Überlegungen hinsichtlich der politischen und dementsprechend auch verfassungsrechtlichen Verwendung von Augmented und Virtual Reality anzustellen. So bildet die naheliegende Frage nach den verfassungsrechtlichen Folgen einer Politisierung von Augmented Reality am Beispiel von Pokémon Go den argumentativen Ausgangspunkt (2.). Sodann sollen die verfassungsrechtlichen Dimensionen von Virtual Reality diskutiert werden: Wo ließe sich Virtual Reality im verfassungspolitischen Prozess und Staatsorganisationsrecht einsetzen? Und wie könnte, würde oder sollte die Verfassungsrechtsordnung darauf reagieren (3.)? 


\section{Augmented Reality: Virtuelle Transformationen des Politischen}

Auf den ersten Blick scheint die Rechts- und Verfassungsordnung auf die Herausforderung, die durch die Augmented Reality von Pokémon Go erzeugt wird, angemessen reagieren zu können (Jacobs et al. 2016; Hilgert und Sümmermann 2016; Prütting und Wilke 2016; Söbbing 2016; Tinnefeld 2016): Soweit Pokémons bzw. Pokéstops so platziert werden, dass Spieler`innen entweder privates Eigentum beeinträchtigen oder die Funktion öffentlicher Einrichtungen - einschließlich Straßen - stören, greifen zivil- und öffentlich-rechtliche Hausrechte und Unterlassungsansprüche, die sowohl gegenüber den einzelnen Spielerinnen und Spielern als auch gegenüber Spielebetreibern rechtlich geltend gemacht werden können. Daneben treten das Polizei- und Sicherheitsrecht: Es dürfen Betretungsverbote und Platzverweisungen ausgesprochen und Ansammlungen aufgelöst werden, wenn sie eine Gefahr für die öffentliche Sicherheit und Ordnung begründen. Darüber hinaus steht die Strafbarkeit des Hausfriedenbruchs im Raum. Schließlich kommen auch Schadensersatzansprüche in Betracht. Kurz: Privatpersonen müssen nicht dulden, dass ihr Eigentum und Besitz aufgrund eines privaten und kommerziellen Spiels analog oder virtuell beeinträchtigt werden; und keine Verwaltung darf es erlauben, dass ihre Funktionen, die dem Gemeinwohl dienen, durch Pokémon Go gestört werden. Damit wird deutlich, dass im Fall von Pokémon Go zunächst keine oder jedenfalls keine wesentlichen Realitätsverschiebungen in der Rechtsordnung stattfinden. Das Verständnis des Hausrechts entwickelt sich als Ausprägung des privaten Eigentums- und öffentlichen Sachenrechts weiter, indem es eine virtuelle Dimension entfaltet: Privatpersonen und Verwaltungen müssen auch keine virtuellen Beeinträchtigungen ihrer Grundstücke bzw. in ihren Gebäuden dulden. So weit, so gut!

Doch was würde sich verändern, wenn Pokémon Go nicht eine Mischung aus Spiel und Kommerz bliebe, sondern politisch würde? Wenn also aus „Pocket Monster“, für das ja „Pokémon“ steht, „Pocket Politics“ würde und die Bürger^innen also anfingen, „Pocket Politics Go“ - „Poképol Go“ - zu „spielen“? Mit dieser Politisierung von Augmented Reality würden sehr viel größere Realitätsverschiebungen in der Rechts- und Verfassungsordnung eintreten. Der öffentliche Raum würde durch politische Augmented Reality transformiert, virtuell vervielfacht und erweitert. Auf der verfassungsrechtlichen Grundlage der Meinungsfreiheit (Artikel 5 Absatz 1 Satz 1 Grundgesetz) könnte sich eine ganz neue, virtuelle Stadtbeschilderung entwickeln. So entstünden neue Formen des Kommentierens und Bebilderns des öffentlichen Raums. Beispielsweise könnten vor Geschäften, 
Unternehmen und Behörden politische Pokémons gesetzt werden, die über Unternehmens- und Behördenpolitiken informieren - bei Unternehmen beispielsweise über Arbeitsbedingungen, bei Behörden über Verwaltungspolitiken. Auch das Versammlungsrecht (Artikel 8 Absatz 1 Grundgesetz) ließe sich mit Hilfe politischer Pokémons sehr viel spontaner und situativer gestalten und wahrnehmen. Allerdings ist eine solche Politisierung von Pokémon Go durchaus ambivalent: Die politischen Pokémons müssen nicht notwendigerweise zu aufgeklärten Zwecken eingesetzt werden. Sie könnten auch beispielsweise dazu dienen, die Wohnungen politischer Gegner zu kennzeichnen, um diese zu terrorisieren. Auch lassen sich mit dieser Technik im Kontext gewalttätiger Demonstrationen beispielweise offene Fluchtwege und neue Sammelplätze in einer Stadt spontan markieren, um Polizeieinsätze strategisch zu umgehen. In rechtlicher Perspektive ist es immer wichtig, auch die pathologischen Anwendungen neuer Kommunikationstechniken im Auge zu behalten. Doch diese Ambivalenz der Medienentwicklung spricht nicht gegen neue Kommunikationstechniken und -formen an sich.

Die legale Entfaltung von Augmented Reality kann dabei den öffentlichen Raum nicht nur um eine neue bildhaft-virtuelle Kommunikationsform bereichern. Vielmehr würde die politische Anwendung von Augmented Reality mit ihrer bildhaft-virtuellen Kommunikation auch zu einer rechtlichen Realitätsverschiebung des öffentlichen Raums beitragen, die sich seit einigen Jahren mit der zunehmenden Kommunikationspflichtigkeit des Privateigentums (Artikel 14 Absatz 1 Grundgesetz) abzeichnet (Siehr 2016): Wenn private Eigentümer einen öffentlichen Raum - ein public forum - eröffnen, müssen sie auch prinzipiell dulden, dass die Bürger`innen in diesem öffentlichen Raum ihre Kommunikationsgrundrechte, also die Meinungs- und Versammlungsfreiheit, wahrnehmen. Diesen Grundsatz hat das Bundesverfassungsgericht vor dem Hintergrund einer zunehmenden Privatisierung des öffentlichen Raums entwickelt. So sind nicht nur die Deutsche Bahn AG als 100\%ige Bundestochter im Hinblick auf Bahnhöfe an die Meinungsund Versammlungsfreiheit gebunden, sondern auch Aktiengesellschaften, die im Mehrheitsbesitz der öffentlichen Hand stehen und zum Beispiel Flughäfen betreiben (BVerfG 2011). Zuletzt hat das Bundesverfassungsgericht auch die Pflicht des privaten Eigentümers eines öffentlichen Platzes bejaht, einen Flashmob zu dulden, der sich gegen die Privatisierung des öffentlichen Raums wendet (BVerfG 2015). Damit verändert sich die rechtliche Kontur des Privateigentums, das zugunsten der Kommunikationsgrundrechte eingeschränkt wird. Diese Entwicklung lässt sich wie folgt verallgemeinern (Kersten 2017a, 228-229): Wenn ein privater Eigentümer ein öffentliches Forum eröffnet, so wird sein analoges, aber auch sein virtuelles Hausrecht mit Blick auf die Kommunikationsfreiheiten der Nutzer^innen dieses öffentlichen Raums eingeschränkt. Es muss ein verhältnismäßiger Ausgleich zwischen der Eigentumsfreiheit einerseits und der Meinungs- und Ver- 
sammlungsfreiheit andererseits in jedem Einzelfall gefunden werden. Diese kommunikationspflichtige Transformation des Eigentums wird durch politische und vor allem aktivistische Augmented Reality einen weiteren Schub erhalten. So ist es beispielsweise möglich, dass die aktuellen Konflikte zwischen Kunstfreiheit (Artikel 5 Absatz 3 Satz 1 Grundgesetz) und Identitätspolitiken um Bilder und andere Kunstwerke in Museen (Kaube 2018; Rauterberg 2018) mittels des virtuellen Kommentierens und Bebilderns in Form von politisierter Augmented Reality im unmittelbaren räumlichen Kontext des Bildes bzw. Kunstwerks im Museum selbst ausgetragen werden. Weil Museen ein Public Forum eröffnet haben, können sie sich insofern grundsätzlich nicht auf ihr virtuelles Hausrecht berufen, um diese virtuellen Kommunikationsformen kategorisch auszuschließen. Vielmehr sehen sich private und öffentliche Museen gerade herausgefordert, die darin liegende politische, aber auch rechtliche Realitätsverschiebung aktiv mitzugestalten. So erweist sich die Rechtsordnung für mediale Realitätsverschiebungen durch Augmented Reality prinzipiell offen.

\section{Virtual Reality: Virtuelle Simulationen des Politischen}

Während Augmented Reality die analoge Lebenswelt virtuell überformt, simuliert Virtual Reality virtuelle Lebenswelten, die sich einerseits von der analogen Welt unterscheiden, zugleich aber auch mit dieser verbunden sind. Diese Verbindung wird vor allem dadurch geschaffen, dass es sich bei Virtual Reality um eine kommunikative Interaktionsform handelt, die durch immersives, unmittelbar körperliches Erleben von Personen geprägt ist (Kelly 2016, 211-217, 226-227, 229; Bailenson 2018, 1-7, 46, 249; Volland 2018, 201-207). Welche rechtlichen Realitätsverschiebungen vollziehen sich hier, wenn sich diese virtuellen Simulationen im politischen Raum entfalten? Um diese Frage $\mathrm{zu}$ beantworten, soll im Folgenden zwischen der grundrechtlichen (3.1.) und der staatsorganisatorischen (3.2.) Sphäre des Politischen unterschieden werden.

\subsection{Grundrechtliche Sphäre des Politischen: Virtuelle Versammlungsfreiheit}

Auch im virtuellen Raum beginnt jede Politik mit der Meinungs- und Versammlungsfreiheit. Menschen finden sich zusammen, um gemeinsam etwas politisch $\mathrm{zu}$ tun, gemeinsam eine politische Meinung zu entwickeln, $\mathrm{zu}$ demonstrieren, 
um Politik zu diskutieren, zu kritisieren und zu beeinflussen. Dies können sie auch als Avatare in virtuellen Räumen. Virtual Reality erleichtert die politische Versammlung von Menschen, die in der analogen Welt räumlich weit voneinander entfernt sind. Welche zentrale Bedeutung Medien und Infrastrukturen gerade für die politische Arbeit spielen, haben schon Karl Marx und Friedrich Engels im Manifest der Kommunistischen Partei mit Blick auf Eisenbahninfrastrukturen beschrieben: Es sind die „wachsenden Kommunikationsmittel, die von der großen Industrie erzeugt werden und die Arbeiter der verschiedenen Lokalitäten miteinander in Verbindung setzen“ (Marx und Engels 1984 [1848], 55). Hier fallen 1848 bereits die zentralen Begriffe, die unser soziales und politisches Denken bis heute bestimmen: „Kommunikationsmittel“, „große Industrie“ und „in Verbindung setzen“ - also Vernetzung. Auch politische Versammlungen in der Virtual Reality bewegen sich in diesem begrifflichen Dreiklang, und gehen zugleich darüber hinaus, indem sie dem Politischen einen vollkommen neuen, virtuellen Raum eröffnen, der körperlich erlebbar ist.

Das Verfassungsrecht ist sich aber gegenwärtig noch unschlüssig, ob es diese Realitätsverschiebung nachvollziehen möchte: Die wohl (noch) herrschende Meinung unter Verfassungsrechtlerinnen und Verfassungsrechtlern hat keine Schwierigkeiten, Bürger*innen, die im virtuellen Raum politisch aktiv werden, die Meinungsfreiheit zuzusprechen. Sie zweifelt jedoch daran, ob man das Versammlungsrecht im virtuellen Raum wahrnehmen kann: Die Versammlungsfreiheit - so das Argument - erfordere es, mit seinem Körper für die eigene Meinung einzustehen (Kingreen und Poscher 2018, 228): Das sei bei einer Versammlung im analogen Raum gewährleistet, nicht aber bei einer Zusammenkunft in der virtuellen Lebenswelt. Diese im Verfassungsrecht noch vorherrschende Auffassung verdient jedoch keine Zustimmung (Kersten 2017c, 198): Schon die klassische Auslegung des Artikel 8 Absatz 1 Grundgesetz nach Wortlaut und Zweck zeigt, dass eine Erstreckung der Versammlungsfreiheit in den virtuellen Raum und damit eine medial begründete rechtliche Realitätsverschiebung problemlos möglich ist. Artikel 8 Absatz 1 Grundgesetz lautet: „Alle Deutschen haben das Recht, sich ohne Anmeldung oder Erlaubnis friedlich und ohne Waffen zu versammeln.“ Eine Versammlung ist nach der klassischen Rechtsprechung des Bundesverfassungsgerichts eine Zusammenkunft von mehreren Personen, die zur Verfolgung eines gemeinsamen Zwecks innerlich verbunden sind (BVerfG 1985, 342-343). Nach der jüngeren Rechtsprechung des Bundesverfassungsgerichts sind Versammlungen „örtliche Zusammenkünfte mehrerer Personen zur gemeinschaftlichen, auf die Teilhabe an der öffentlichen Meinungsbildung gerichteten Erörterung oder Kundgebung“ (BVerfG 2002 [2001], 104; ferner BVerfG 2011, 250). Doch aus der definitorischen Anforderung einer „örtlichen Zusammenkunft“ ergibt sich keineswegs zwangsläufig, dass 
sich der Ort der gemeinsamen Versammlung notwendigerweise in der analogen Welt befinden müsste. Es kann auch ein virtueller Raum gewählt werden. Bisher haben sich Menschen in der Regel im öffentlichen Straßenraum versammelt. Dies bedeutet allerdings nicht, dass der grundrechtliche Schutz des Artikels 8 Absatz 1 Grundgesetz allein auf diese tradierten Versammlungsorte und zugleich auch auf die überkommenen Versammlungsformen der analogen Welt beschränkt wäre. Vielmehr betont das Bundesverfassungsgericht in ständiger Rechtsprechung: Die Versammlungsfreiheit ist als „Ausdruck gemeinschaftlicher, auf Kommunikation angelegter Entfaltung“ (BVerfG 1985, 343; ferner BVerfG 2002 [2001], 104) geschützt. Es unterstreicht darüber hinaus auch die Offenheit des durch Artikel 8 Absatz 1 Grundgesetz vermittelten Schutzes für vollkommen neue Formen des Sich-Versammelns (BVerfG 1985, 343). Dieser kommunikative Sinn- und Handlungszusammenhang kann aber gerade auch durch das gemeinschaftliche SichVersammeln verwirklicht werden, wenn Bürger*innen als Avatare im virtuellen Raum zu einem gemeinsamen politischen Zweck verbunden sind. Damit ist der Schutz der Versammlungsfreiheit nicht auf physische Zusammenkünfte in der analogen Welt beschränkt. Vielmehr erstreckt sich die Gewährleistung des Artikel 8 Absatz 1 Grundgesetz auch auf Versammlungen in der virtuellen Lebenswelt. Die damit verbundene Realitätsverschiebung des Rechts ist ohne eine Rechtsänderung durch Verfassungsauslegung möglich. Diese Realitätsverschiebung sollte auch für die oben zitierte herrschende Meinung nachvollziehbar sein, die hinsichtlich der Versammlungsfreiheit auf die körperliche Präsenz der Büger^innen abstellt. Denn eine solche körperliche Präsenz wird ja gerade auch im virtuellen Raum hergestellt. Wir sind mit unserem Avatar körperlich verbunden. Virtual Reality ist ein immersives, interaktives körperliches Erlebnis. Dies führt uns hinsichtlich der Gewährleistung der Versammlungsfreiheit, aber auch darüber hinaus vor Augen, dass eine strikte Unterscheidung zwischen analoger und virtueller Realität und somit zwischen nur analogen oder nur virtuellen Grundrechtsgewährleistungen in der „nächsten Gesellschaft“ keinen Sinn (mehr) macht.

Mit dieser grundrechtlichen Realitätsverschiebung sind jedoch keineswegs alle rechtlichen Probleme „gelöst“, sondern es stellen sich - ganz im Gegenteil - vollkommen neue Herausforderungen. Diese lassen sich beispielhaft an der Frage erörtern, welche Anforderungen an den Grundrechtsträger in der Virtual Reality zu stellen sind. Dies mag zunächst verblüffen, da selbstverständlich alle Büger`innen das Grundrecht der Versammlungsfreiheit im analogen und eben auch im virtuellen Raum wahrnehmen können. Bei der Inanspruchnahme der Versammlungsfreiheit im analogen Raum ist die Beantwortung dieser Frage problemlos möglich: Die Büger`innen, die körperlich anwesend sind, werden durch Artikel 8 Absatz 1 Grundgesetz geschützt. Dies ist im virtuellen Raum zwar grundsätzlich genauso. Nur weiß man in der virtuellen Lebenswelt unter 
Umständen nicht genau, wer überhaupt „wirklich“ anwesend ist (Scheiner 2018, 55). Unsere Avatare müssen kein getreues Abbild unserer selbst sein. Dies ist in erster Linie kein Problem des sogenannten „Vermummungsverbots“, das von den Versammlungsgesetzen für Versammlungen in der analogen Lebenswelt vorgesehen ist (vgl. beispielsweise $\S 17 a$ Absatz 2 Versammlungsgesetz). Denn grundsätzlich wird das Recht, sich auch „vermummt“ zu versammeln, durch Artikel 8 Absatz 1 Grundgesetz geschützt und bedarf angesichts des aktuellen Bedeutungswandels von Anonymität in der analogen und erst recht in der virtuellen Welt einer vollkommen neuen, liberalisierenden Bewertung (Kersten 2017c). Doch das Problem der Grundrechtssubjektivität stellt sich in der virtuellen Lebenswelt noch grundlegender, da wir hier auch Avataren begegnen, hinter denen kein menschliches Subjekt, sondern Künstliche Intelligenzen stehen, ohne dass wir dies in der kommunikativen Interaktion ohne weiteres erkennen können. Auch wenn nicht von vornherein ausgeschlossen ist, dass nichtmenschliche Entitäten und damit auch Avatare einer Künstlichen Intelligenz über Grundrechtsschutz verfügen können (Kersten 2017b, 2019, 444-450): Prinzipiell müssen Grundrechtsträger als solche zu erkennen sein oder sich als solche zu erkennen geben.

Dies führt zu einem strukturellen Grundproblem der virtuellen Lebenswelt, das weit über die Grundrechtsgewährleistung hinausgeht: Wann müssen menschliche Personen und nichtmenschliche Entitäten in der virtuellen Lebenswelt unterscheidbar sein? Eine solche Unterscheidung ist beispielsweise für die Gewährleistung der Konsistenz zwischenmenschlicher Interaktion nach Artikel 22 Absatz 1 Datenschutzgrundverordnung in der virtuellen Lebenswelt notwendig. Nach dieser Regelung hat jede Person das Recht, nicht einer ausschließlich auf einer automatisierten Verarbeitung beruhenden Entscheidung unterworfen zu werden, die ihr gegenüber rechtliche Wirkung entfaltet oder sie in ähnlicher Weise erheblich beeinträchtigt. Dies bedeutet beispielsweise für das Arbeitsrecht: Menschen und Roboter können in der analogen Lebenswelt und menschliche und „nichtmenschliche“ Avatare in der virtuellen Lebenswelt durchaus zusammenarbeiten, wenn eine ausdrückliche Einwilligung des betroffenen menschlichen Arbeitnehmers vorliegt (Artikel 22 Absatz 2 Buchstabe c Datenschutzgrundverordnung; Giesen 2018). Dies erfordert aber zugleich, dass hinsichtlich der Kompetenz, rechtlich verbindliche Entscheidungen für Menschen zu treffen, Menschen und Maschinen nicht nur in der analogen, sondern insbesondere auch in der virtuellen Arbeitswelt prinzipiell unterscheidbar sein müssen (grundsätzlich Martini 2017b, 1020; Lenzen 2018, 230).

Ein solcher humaner Vorbehalt gilt grundsätzlich auch für die Inanspruchnahme des Grundrechtsschutzes: Wer für sich und seinen Avatar die Versammlungsfreiheit in der Virtual Reality in Anspruch nehmen möchte, muss sich 
zumindest (derzeit) als menschlicher Akteur ausweisen, selbst wenn er seine personelle Identität angesichts der sich aktuell wandelnden Bedeutung von Anonymität nicht notwendigerweise vollkommen offenlegen muss. So bildet die humane Selbstlegitimation - „Ich bin ein Mensch“/„Ich bin kein Roboter“ gegenwärtig (noch) die Basis des Grundrechtsschutzes. Damit geht nicht automatisch eine flächendeckende Kennzeichnungspflicht für nichtmenschliche Akteure in der virtuellen Kommunikation einher, deren rechtliche und faktische Durchsetzungsmöglichkeiten fraglich sind. Ein virtuelles Verwirrspiel um richtige und falsche Kennzeichnungen des (Nicht-)Humanen wäre die wahrscheinliche Folge einer umfassenden Kennzeichnungspflicht für Roboter und Künstliche Intelligenzen. Ob ein humaner Vorbehalt für die Inanspruchnahme von Grundrechten und auch für das rechtserhebliche Entscheiden in einer virtuellen Lebenswelt, die zunehmend von subsymbolisch trainierter Künstlicher Intelligenz geprägt sein wird, wirklich eine Zukunft hat, ist mehr als nur zweifelhaft (Kersten 2019, 444-450). Doch wir machen mit diesem humanen Vorbehalt nur unsere ersten tastenden Schritte in die „nächste Gesellschaft“, deren Verfassung große Gestaltungsspielräume für weitere Realitätsverschiebungen lässt.

\subsection{Staatliche Sphäre des Politischen: Virtualität der drei Gewalten}

Die drei Gewalten - Legislative, Exekutive und Judikative (Artikel 20 Absatz 2 und 3 Grundgesetz) - werden auch in der „nächsten Gesellschaft“ nicht vollständig von der analogen in die virtuelle Lebenswelt wechseln; oder vorsichtiger formuliert: Dies erscheint aus heutiger Perspektive jedenfalls schwer vorstellbar. Ungeachtet dessen könn(t)en die drei Gewalten aber schon heute - wenn auch in sehr unterschiedlichem Maß - Virtual Reality nutzen, um ihre verfassungsrechtlichen Funktionen zu erfüllen.

\subsubsection{Exekutive}

Die Exekutive - Regierung und Verwaltung - setzt bereits Virtual Reality für die Erfüllung ihrer Aufgaben ein: Militär und Polizei verwenden Virtual Reality für Trainingszwecke sowie für die psychologische Bewältigung von Traumata, die sich nach militärischen und polizeilichen Einsätzen entwickeln können (Bailenson 2018, 41-42, 136-149, 251-252; zu Recht ambivalent Lanier 2018, 295-298). Mit pädagogischer Zielsetzung kann Virtual Reality in staatlichen Schulen, Universitäten und Museen genutzt werden (Bailenson 2018, 42; Kohle 
2018; Volland 2018, 167). Ein weites Anwendungsfeld eröffnet sich im Planungsrecht und hier vor allem in der Öffentlichkeitsbeteiligung, wenn etwa die ökologischen Folgen von Planungen im Städtebau oder von Infrastrukturen, Straßen, Bahnhöfen und Flughäfen aufgezeigt und für die Bürger- und die Behördenbeteiligung aufbereitet werden. Virtual Reality vermag hier, Planungsalternativen einschließlich der jeweiligen ökologischen Folgen zu veranschaulichen. In diesem Zusammenhang können Manipulationen durch die Standardisierung und Offenlegung der verwendeten Software vorgebeugt werden. Sollte es trotzdem zu Planungsfehlern kommen, lassen sich diese nach einem entsprechenden Planmonitoring korrigieren. Im Grundsatz sind im Rahmen der Exekutive auch sehr weitgehende Virtualisierungsstrategien denkbar. Das Konzept von Electronic Government (eGovernment) lässt sich zu Virtual Government (vGovernment) weiterentwickeln. Realitätsverschiebungen von Bürokratien aus der analogen in die virtuelle Lebenswelt sind ebenso denkbar wie Behörden und Rathäuser in der Virtual Reality. Allerdings gestalten sich die Aufgaben der Exekutive für unsere Gesellschaft mit der Gewährleistung von physischer und sozialer Sicherheit, von Daseinsvorsorge, Infrastrukturen und Umweltschutz so umfassend, dass Regierung und Verwaltung auch in Zukunft sowohl in der analogen als auch in der virtuellen Welt präsent sein müssen. Soweit sich die Exekutive aber in der Virtual Reality entfaltet, stellen sich auch hier die beiden zentralen Fragen, die uns bereits im Kontext der virtuellen Versammlungsfreiheit mit Blick auf die Erkennbarkeit und die rechtsverbindlichen Entscheidungskompetenzen Künstlicher Intelligenzen beschäftigt haben (3.1.).

Inwieweit muss der Staat - also erstens - in der Virtual Reality erkennbar sein? Wenn staatliche Verwaltungen in der Virtual Reality aktiv werden, haben sie sich auch prinzipiell als staatliche Akteure zu erkennen zu geben. Dies ist insbesondere dann der Fall, wenn die Polizei für Sicherheit in der virtuellen Lebenswelt sorgen soll (Ingold 2017a). Das Bundesverfassungsgericht hat in seiner Entscheidung zu Online-Durchsuchungen vom 27. Februar 2008 der Verwaltung einen relativ großen Spielraum im Hinblick auf ein anonymes bzw. verdecktes Auftreten in der Netzkommunikation gelassen (BVerfG 2008, 340-346). Doch dies ist zu Recht auf Kritik gestoßen. Die Kompetenzen von Beamtinnen und Beamten, in der digitalen Kommunikation und in der Virtual Reality offen, anonym oder verdeckt zu agieren, müssen gesetzlich geregelt werden. Dabei ist vor allem dem Persönlichkeitsrecht der Büger^innen Rechnung zu tragen, das insbesondere das Recht auf informelle Selbstbestimmung (BVerfG 1984 [1983], 41-45) sowie das Recht auf Gewährleistung der Vertraulichkeit und Integrität informationstechnischer Systeme (BVerfG 2008, 302-306) umfasst.

Inwieweit darf der Staat - zweitens - in der Virtual Reality seine rechtsverbindlichen Entscheidungskompetenzen durch Künstliche Intelligenzen 
wahrnehmen? Müssen im virtuellen Rathaus hinter den Avataren „reale“ Menschen stehen oder dürfen auch Künstliche Intelligenzen den Staat repräsentieren? Weitgehend unproblematisch können Künstliche Intelligenzen zunächst in die Beratung für Bürgerinnen und Bürgern durch die Verwaltung einbezogen werden (Martini 2017a, 453). Allerdings ist die geltende Rechtsordnung sehr zurückhaltend, wenn es darum geht, dass Künstliche Intelligenzen rechtsverbindliche Entscheidungen gegenüber den Bürgerinnen und Bürgern treffen: Ein Verwaltungsakt kann vollständig durch automatische Einrichtungen erlassen werden, sofern dies durch Rechtsvorschrift zugelassen ist und weder ein Ermessen noch ein Beurteilungsspielraum besteht ( $\S 35$ a Verwaltungsverfahrensgesetz). Mit diesem humanen Vorbehalt für die wertungsbedürftige Ausfüllung von Beurteilungsspielräumen und die Alternativen wägende Ermessensausübung will das Verwaltungsverfahrensgesetz eine restriktiv vorbeugende Regelung treffen, um dem Persönlichkeitsrecht der Büger^innen zu genügen (Martini und Nink 2017, 2). Doch auch hier wird die normative Kraft der Kommunikationstechnik- und Medienentwicklung weiteren Veränderungsdruck auslösen. Denn subsymbolisch trainierte Künstliche Intelligenzen können vor allem eines: Muster und Strukturen erkennen (Ramge 2018, 43-49, 61). Gerade dies prägt aber auch die Ausfüllung verwaltungsrechtlicher Beurteilungsspielräume und die Ermessenausübung der Verwaltung. Vor diesem Hintergrund ist es wahrscheinlich, dass Künstliche Intelligenzen insbesondere in der Massenverwaltung zunächst lernend parallel zur menschlichen Sachbearbeitung mitlaufen und diese ab einen bestimmten Punkt vollkommen eigenständig übernehmen. Hinsichtlich atypischer Fallgestaltungen könnte man an einem humanen Entscheidungsvorbehalt festhalten oder an hybride Entscheidungsverfahren von menschlicher Sachbearbeitung und Künstlicher Intelligenz denken. Auf diese Weise würde die enge Verbindung der Entwicklung von Künstlicher Intelligenz und Virtual Reality (Kelly 2016, 229-230) nach einer entsprechenden Rechtsänderung die Möglichkeit eröffnen, auch einem intelligenten nichtmenschlichen Avatar in der virtuellen Behörde rechtsverbindliche Entscheidungskompetenzen gegenüber Bürgerinnen und Bürgern zu übertragen. Mit anderen Worten: Die normative Kraft der Kommunikationstechnik- und Medienentwicklung wird hier eher früher als später zu weiteren rechtlichen Realitätsverschiebungen führen.

\subsubsection{Judikative}

Die Judikative steht der Anwendung von Virtual Reality im Vergleich zur Exekutive sehr viel reservierter gegenüber. Die Frage, ob und inwiefern Virtual Reality in der gerichtlichen Beweiserhebung eingesetzt werden kann bzw. 
sollte, wird vor allem in den USA prozessrechtlich kritisch hinterfragt: Ist Virtual Reality nicht „too real“ - also „zu real“ - für Geschworene und Richter^innen, wenn es beispielsweise um einen virtuell rekonstruierten Tatort geht (Feigenson 2006; ferner Joseph 1996)? Virtual Reality vermittelt eine körperlich umfassende Erfahrung. Sie könnte deshalb überschießende Realitätserlebnisse generieren, ja eine vollkommen eigenständige Realität für das Gericht erzeugen, die mit der Realität am Tatort zur Tatzeit überhaupt nichts zu tun hat. Darüber hinaus wird die virtuelle Rekonstruktion eines Tatorts regelmäßig auch auf Zeugenaussagen beruhen, die selbst wiederum selektiv und ungenau sind, wenn nicht sogar schlicht falsch sein können. Diese Unsicherheit bildet Virtual Reality aber gerade nicht ab. Schließlich bleibt die virtuelle Realität selektiv, weil beispielsweise Temperaturen, Gerüche und andere Sinneseindrücke nicht oder jedenfalls nicht vollständig abgebildet werden. Der gerichtliche Streit um die Würdigung von einzelnen Zeugenaussagen würde sich in eine prozessuale Auseinandersetzung um die „Realität“ des virtuellen Beweises verlagern. Was wäre damit gegenüber einer auf dem Grundsatz der Unmittelbarkeit der Beweiswürdigung beruhenden Tatsachenfeststellung im Gerichtsverfahren gewonnen? Nicht wirklich viel. Damit scheidet die Einführung von Virtual Reality in ein Gerichtsverfahren allerdings nicht vollkommen aus. Es bliebe die Möglichkeit, Virtual Reality im Rahmen des Sachverständigenbeweises in eine Verhandlung einzubringen. Der Sachverständigenbeweis ist immer dann angezeigt, wenn der Richterin oder dem Richter die notwendige Sachkunde für die Einschätzung eines Sachverhalts fehlt (BGH 2015, 1602). Bereits heute werden Computersimulationen von Sachverständigen, die der Richterin bzw. dem Richter auch die Simulationstechnik erläutern, als Grundlage für die gerichtliche Würdigung von Verkehrsunfall- oder Brandhergängen im Prozess verwendet (OLG Hamm 2004; OLG Jena 2012 [2011]; OLG München 2016).

Einen Schritt weiter führt die grundsätzliche Frage, ob Gerichte insgesamt in der Virtual Reality verhandeln könnten. In einer virtuellen Gerichtsbarkeit würden die Parteien und die Richter`innen als Avatare in einem virtuellen Gerichtssaal tagen, um beispielsweise Zeit und Raum, Personal und Kosten zu sparen. Allerdings ist dies gegenwärtig nicht möglich. Gerichtsverfahren finden in einem analogen Gerichtssaal statt, der über Öffentlichkeit, Mündlichkeit und Unmittelbarkeit der Anschauung der Beweise die mediale Grundlage für das Urteil im Namen des Volkes schafft. Die Gerichtsverfassung hält grundsätzlich an dieser analogen Medienordnung fest und lässt Ton- und Filmaufnahmen nur ausnahmsweise zu (§ 169 Gerichtsverfassungsgesetz; § 17a Bundesverfassungsgerichtsgesetz). Darüber hinaus erlaubt die Zivilprozessordnung die Verhandlung im Wege der Bild- und Tonübertragung, wenn sich Parteien, Prozessvertreter, Zeugen oder Sachverständige an einem anderen Ort aufhalten (§ 128a Zivilpro- 
zessordnung). Dabei ist die Verhandlung bzw. Vernehmung zeitgleich in Bild und Ton an diesem Ort und in dem eigentlichen Gerichtssaal zu übertragen; und diese Übertragung darf nicht aufgezeichnet werden, um den analogen Inbegriff der Hauptverhandlung nicht medial zu gefährden. Die Strafprozessordnung kennt eine ähnliche Regelung, um beispielsweise Zeugenschutz zu gewährleisten, wobei hier allerdings eine Aufzeichnung zur Beweissicherung möglich ist (§ 247a Absatz 1 Strafprozessordnung). Das Prozessrecht erweist sich damit bisher medial als nur sehr eingeschränkt lernfähig, um die Rechte der Bürger`innen zu schützen und effektiven Rechtsschutz zu gewährleisten. Zugleich ist es aber auch nicht von vornherein kategorisch ausgeschlossen, Mündlichkeit und Unmittelbarkeit einer Gerichtsverhandlung im virtuellen Raum herzustellen, gerade wenn es allein um Rechts- und nicht um Sach- und Beweisfragen geht. Wenn sich das Prozessrecht auf den Weg zum virtuellen Gerichtssaal begeben möchte, würde dieser zunächst über die freiwillige Gestaltung virtueller Streitbeilegung im ziviloder wirtschaftsrechtlichen Kontext führen, um Erfahrungen mit dieser Form der Realitätsverschiebung zu sammeln.

\subsubsection{Legislative}

Bleibt die Legislative - der Deutsche Bundestag - und damit das in unserer Verfassungsordnung einzig demokratisch unmittelbar durch das Volk in Form von Wahlen legitimierte Staatsorgan (Artikel 38 Grundgesetz). Die demokratische Repräsentation des Volkes im Parlament beruht ebenfalls auf einer Medienverfassung - konkreter: auf einem Medienmix, in dem sich die einzelnen Schritte der Medienentwicklung ergänzen und überlagern: die durch analoge und digitale Medien sowie Rundfunk und Fernsehen geprägte demokratische Öffentlichkeit (Artikel 5 Absatz1, Artikel 8 Absatz1, Artikel 9 Absatz1, Artikel 21 Absatz 1 Grundgesetz), die Stimmabgabe auf papierenen Wahlzetteln (Artikel 38 Absatz 1 Grundgesetz), die öffentliche Stimmmauszählung und Feststellung des Wahlergebnisses (Artikel 20 Absatz 1 - Absatz 3 Grundgesetz), die Konstitution des neu gewählten Bundestags im Parlamentsgebäude (Artikel 39 Absatz 2 Grundgesetz), das gesprochene Wort im öffentlichen Plenum des Bundestags (Artikel 42 Absatz 1 Grundgesetz) in Form der freien Rede und Gegenrede ( $§ 28$, $\S 33$ Geschäftsordnung des Deutschen Bundestags) und das wahrheitsgetreue geschriebene Wort (Artikel 42 Absatz 2 Grundgesetz) in Form von stenografischen Plenarprotokollen in Verbindung mit parlamentarischen Drucksachen. Die Medienverfassung der parlamentarischen Repräsentation wurde ursprünglich und wird bis heute durch das gesprochene und geschriebene Wort geprägt. Sie hat jedoch zugleich auch medial dazugelernt und sich für neue Medienent- 
wicklungen geöffnet: für Zeitungen und Presse im neunzehnten Jahrhundert, für den Rundfunk ab den 1920er Jahren, für das Fernsehen ab den 1950er Jahren und - entgegen vieler anderslautender Diagnosen - auch für die sozialen Medien und das Web 2.0 (Kersten 2016, 2017a, 159-188). Es muss also am Maßstab des Grundgesetzes immer wieder darüber entschieden werden, ob neue Kommunikationstechniken in die Medienverfassung der demokratischen Repräsentation integriert werden können oder ob an dem überkommenen Medieneinsatz festzuhalten ist, um die demokratische Funktion des Parlaments zu gewährleisten. So hat das Bundesverfassungsgericht beispielsweise entschieden, dass Onlinewahlen zum Deutschen Bundestag (derzeit) verfassungsrechtlich nicht möglich sind, weil sie gegen den ungeschriebenen Verfassungsgrundsatz der Öffentlichkeit und Nachvollziehbarkeit der Wahl verstoßen, den die Karlsruher Richter^innen aus dem Demokratie-, Rechtsstaats- und Republikprinzip (Art. 20 Abs. 1-3 GG) hergeleitet haben (BVerfG 2010 [2009]): Wir Bürger^innen müssen diesen zentralen demokratischen Legitimationsakt unseres politischen Gemeinwesens selbst nachvollziehen können. Demgegenüber bereichert die Einführung von Online-Petitionen den parlamentarischen Prozess (Artikel 17 Grundgesetz), indem sie die demokratische Teilhabe von Bürgerinnen und Bürgern an der Rechtsetzung fördern (Kersten 2016, 330-331). Deshalb lautet auch die entscheidende Frage hinsichtlich Virtual Reality, ob und - wenn ja - welchen funktionalen Anschluss diese Kommunikationstechnik an die repräsentative Demokratie gewinnen kann.

Bei der Bestimmung der Möglichkeiten und Grenzen eines virtuellen Parlamentarismus mag zunächst ein Gedankenexperiment weiterhelfen, das an das Modell des „direkten Parlamentarismus“ (Plaum 2013, 148-153) anknüpft: Wir stellen uns ein Parlament in der Virtual Reality vor, in dem alle Bürger`innen als Avatare anwesend sein können, um - gegebenenfalls per Zufall ausgewählt - das Wort zu ergreifen und mit abzustimmen. Wäre das demokratischer als die Repräsentation des Volkes durch die Anwesenheit der - aktuell - 709 Abgeordneten im Reichstagsgebäude? Doch das ist nicht der Fall. Denn die parlamentarische Repräsentation des Volkes zielt - wie sie in Artikel 20 Absatz 2 und Artikel 38 Absatz 1 Grundgesetz verfassungsrechtlich vorgesehen ist nicht auf die reale Spiegelung des realen Volkes in einem realen Parlament. Vielmehr geht es in der parlamentarischen Demokratie des Grundgesetzes um die virtuelle Repräsentation des ganzen Volkes in einem realen Parlamentsgebäude, das die demokratische Institution des Parlaments symbolisiert, also die verfassungsrechtliche Vermittlung zwischen dem unmittelbar gewählten einzelnen Abgeordneten, dem parlamentarischen Plenum und der Repräsentation des ganzen Volkes. Mit anderen Worten: Die demokratische Repräsentation im Parlament beruht gerade auf der Nichtidentität von realem Volk und parlamentarischem Plenum. Deshalb wird auch einer Versammlung potentiell aller 
Bürger*innen als Avatare in der Virtual Reality gerade keine demokratische Repräsentation des Volkes gelingen. Der Versuch, das reale Volk virtuell zu versammeln, scheitert bereits faktisch: Schon aufgrund regelmäßiger Ausbildungsund Arbeitszeiten verfügen die meisten Bürger`innen nicht über die Zeit, sich dauerhaft in einem virtuellen Repräsentationsraum aufzuhalten, zu diskutieren und abzustimmen. Schon das würde zu einer eklatanten Verzerrung des demokratischen Prozesses führen, von dem dadurch eröffneten unmittelbaren Einfluss von Interessenverbänden und Lobbyisten auf den demokratischen Diskussions- und Entscheidungsprozess ganz $\mathrm{zu}$ schweigen. Dies bedeutet: Eine demokratische Repräsentation des realen Volkes in der Virtual Reality wird faktisch, politisch und rechtlich nicht gelingen.

Dies heißt jedoch nicht, dass sich Virtual Reality nicht in die Medienverfassung der demokratischen Repräsentation im Parlament integrieren ließe. Virtual Reality könnte beispielsweise die Möglichkeit von Mehrfachpräsenzen der Abgeordneten eröffnen: Mandatsträger^innen könnten mittels Virtual Reality beispielsweise gleichzeitig im Plenum und in einem Ausschuss des Bundestags anwesend sein oder im Plenum des Bundestags in Berlin, bei der Europäischen Union in Brüssel und bei der UN in New York. Im Gegensatz hierzu wird aber mit Hinweis auf die digitale und virtuelle Medienentwicklung für ein Prinzip der physischen Anwesenheit der Abgeordneten im Bundestag geworben, um die demokratische Repräsentation des Volkes im Parlament für die Bürger^innen anschaulicher und erlebbarer zu gestalten (Schönberger 2016). Doch so verständlich dieses Anliegen verfassungspolitischer Anschaulichkeit zur Stärkung der demokratischen Integration der Bürgerinnen auch sein mag: Die Forderung der Präsenz realer Abgeordnetenkörper im Parlament kann sich medial nicht von einem Repräsentationsverständnis lösen, das sich (noch) ganz „im Schatten des Königs“ bewegt (Manow 2015). Die demokratische Repräsentation geht aber im Verfassungsstaat über die revolutionäre Sukzession vom politischen Körper des gestürzten Königs zum politischen Körper des souveränen Volkes und seines Parlaments hinaus. Ohne seinen revolutionären Ursprung in der amerikanischen und französischen Revolution zu verleugnen, entfaltet der Verfassungsstaat den demokratischen Willen des Volkes in verfassungsrechtlichen Verfahren: „Demokratien sind nicht repräsentativ, sondern expressiv“ (Möllers 2008, 28; ferner 2013, 132-134). Die Pointe dieses demokratischen Expressionismus besteht im Verfassungsstaat jedoch gerade darin, dass seine zentrale politische Ausdruckform in der parlamentarischen Repräsentation des Volkes liegt. Diese ist jedoch nicht (mehr) Ausdruck einer Körperpolitik, die das monarchische Ancien Régime prägte, sondern eine verfassungsförmige und deshalb körperlose Verfahrenspolitik, die den demokratischen Willen der Bürger*innen entfaltet. Es kommt für die demokratische Repräsentation des ganzen 
Volkes im Deutschen Bundestag im Sinn des Artikel 38 Absatz 1 Satz 2 Grundgesetz folglich nicht auf die körperliche Präsenz der 709 Abgeordneten an, sondern auf die Entfaltung des demokratischen Willens des Volkes in Form der parlamentarischen Verfahren, die das Grundgesetz, das Abgeordnetengesetz und die Geschäftsordnung des Deutschen Bundestags vorsehen. Albert Ingold (2017b, 530-533) hat dies auf die treffende Formel gebracht, dass es der demokratischen Repräsentation des Volkes nicht auf das „Präsens einer Präsenz“ von Abgeordneten im Plenarsaal, sondern auf die „Präsenz eines Präsens“ ankommt, also auf die verfahrensrechtliche Entfaltung (Präsenz) des demokratischen Willens des Volkes (Präsens). Bei der demokratischen Repräsentation handele es sich - so Ingold (2017b, 532) - „um einen prozedural-performativ zu vergegenwärtigenden und erlebbaren Prozess“, der sich - in Anknüpfung an Ingolfur Blühdorns Demokratietheorie (2013, 177-178) - durch seinen simulativen Charakter auszeichne. Dieses Verständnis demokratischer Repräsentation erweist sich insbesondere auch für eine dynamische Kommunikationstechnik- und Medienentwicklung als unmittelbar anschlussfähig. Es reflektiert damit den Grundgedanken, dass insbesondere die repräsentative Demokratie nicht den Anschluss an die gesellschaftliche Kommunikation und damit an den gesellschaftlichen Mediengebrauch verlieren darf, sondern aus jedem Medienwandel neue demokratische Legitimationspotenziale schöpfen kann und muss, um die demokratische Funktionsfähigkeit parlamentarischer Repräsentation zu stärken. Dies entspricht auch dem Befund, dass die aktuelle Medienverfassung des parlamentarischen Regierungssystems durch einen Medienmix gekennzeichnet ist, in dem sich inzwischen eine Vielzahl analoger, digitaler und virtueller Kommunikationsformen ergänzen und überlagern. In diese Medienverfassung kann sich auch Virtual Reality einpassen, beispielsweise in Form virtueller Mehrfachpräsenzen von Abgeordneten.

Diese Frage nach einem virtuellen Parlament hat sich unmittelbar zu Beginn der Corona-Krise im März 2020 gestellt: Der Bundestag besteht aus 709 Abgeordneten, die zu Plenardebatten und Ausschusssitzungen im Reichstagsgebäude zusammenkommen. Dabei kann aufgrund der räumlichen Gegebenheiten das Distanzgebot von 1,5 Metern nicht eingehalten werden, um Infektionen zu vermeiden. Wie kann unter diesen Umständen die Funktionsfähigkeit des Parlaments in der Pandemie gewährleistet werden? Der Bundestag behalf sich zunächst rein praktisch, indem er seine Beschlussfähigkeit auf ein Viertel seiner Abgeordneten herabsetzte (§ 126a Absatz 1 Geschäftsordnung des Deutschen Bundestages). Auf dieser Grundlage haben die Fraktionsvorsitzenden vereinbart, dass jede Fraktion nur so viele Abgeordnete in das Plenum entsendet, dass die Mehrheitsverhältnisse gewahrt sind (sogenanntes Pairing-Verfahren; vgl. C. Schönberger und S. Schönberger 2020). Auf diese Weise wurde ein informelles Notparlament geschaffen, das gegebenenfalls aus 177 Abgeordneten besteht, während 532 Abgeordnete frei- 
willig oder aufgrund von Fraktionsdisziplin auf ihre Mandatsausübung verzichten. Dies ist jedoch keine Praxis, die auf Dauer mit der Gewährleistung des freien Mandats und des demokratischen Repräsentationsprinzips (Artikel 38 Absatz 1 Satz 2 GG) vereinbar ist. Deshalb hat Bundestagspräsident Wolfgang Schäuble in der Corona-Krise vorgeschlagen, entweder ein Notparlament ausdrücklich im Grundgesetz zu verankern oder über Formen eines virtuellen Parlamentarismus nachzudenken (Roßmann und Mascolo 2020). Doch wenn man bereits ist, auch nur einfachste Kommunikationstechniken für die Gestaltung von Parlamentssitzungen zu verwenden, kann man getrost auf die verfassungspolitisch zweifelhafte Installierung eines Notparlaments verzichten. Dies hat das britische Unterhaus gezeigt, indem es in der Corona-Krise in Form einer medialen „Hybrid-Sitzung“ zusammenkam (Buchsteiner 2020): In der ersten Prime Minister's Question Time nach der Osterpause 2020 waren im Unterhaus „nur“ Außenminister Dominic Raab, der den an Covid-19 erkrankten britischen Premierminister Boris Johnson vertrat, der Oppositionsführer Keir Starmer sowie der Speaker und etwa 20 weitere Abgeordnete körperlich anwesend, die in entsprechenden Abständen im Parlamentssaal verteilt saßen. Die meisten Abgeordneten waren aus ihren Privatwohnungen per Videokonferenz zugeschaltet und auf einem großen Bildschirm im Parlament zu sehen.

Solche medialen „Hybrid-Sitzungen“ wären auch im Deutschen Bundestag möglich. Zwar geht die Staatsrechtslehre und Staatspraxis bisher überwiegend davon aus, dass die Abgeordneten körperlich im Plenarsaal anwesend sein müssen (Deutscher Bundestag-Wissenschaftliche Dienste 2020, 3). Verfassungsrechtlich zwingend ist diese Auffassung aber nicht. Wenn das Grundgesetz von dem „Zusammentritt“ (Artikel 39 Absatz 1 Satz 2 und Absatz 2 GG) und den „Sitzungen“ des Bundestags (Artikel 39 Absatz 3, Artikel 42 Absatz 1 Satz 3 und Absatz 3 Grundgesetz) spricht, ist dies von seinem Wortlaut hinreichend offen, um hierunter auch die „virtuelle“ Anwesenheit von Abgeordneten $\mathrm{zu}$ verstehen. Die gleiche mediale Offenheit prägt auch die öffentliche Verhandlung (Artikel 42 Absatz 1 Satz 1 GG) oder die Äußerung im Bundestag oder in einem seiner Ausschüsse (Artikel 46 Absatz 1 Satz 1 GG). Auch die Verwendung des Begriffs des „Saals“ in der Geschäftsordnung des Deutschen Bundestages lässt sich in das Konzept einer medialen „Hybrid-Sitzung“ einpassen. Wenn die Redner/innen von den dafür bestimmten Saalmikrofonen oder vom Rednerpult aus sprechen sollen (§ 34 Geschäftsordnung des Deutschen Bundestages), schließt dies die Möglichkeit ein, sich über eine Video- oder digitale Bildübetragung im Saal zu äußern. Das „Rednerpult“ lässt sich ebenfalls virtuell oder gegebenenfalls sogar symbolisch verstehen. Ein „Saalverweis“ kann auch im Rahmen einer Video- oder digitalen Bildübertragung problemlos erfolgen (§ 38 Absatz 1 Satz 1 Geschäftsordnung des Deutschen Bundestages). Das Glei- 
che gilt für eine Abstimmung per Handzeichen bzw. durch Aufstehen oder Sitzenbleiben (§ 48 Absatz 1 Geschäftsordnung des Deutschen Bundestages). Nur komplexere Abstimmungsverfahren wie die geheime Stimmabgabe (§ 49 Geschäftsordnung des Deutschen Bundestages), die namentliche Abstimmung (§ 52 Geschäftsordnung des Deutschen Bundestages) oder der „Hammelsprung“ (§ 51 Absatz 2 Geschäftsordnung des Deutschen Bundestages) müssten virtuell ausgestaltet und entsprechend rechtlich neu geregelt werden, weil sie mit den „Wahlzellen“, der „Türzählung“ und der Sammlung von „Abstimmungskarten“ in „Wahlurnen“ durch analoge Abstimmungsmedien geprägt sind. Diesen Weg in Richtung eines „Virtuellen Parlamentarismus“ sollte der Deutsche Bundestag auch über die Corona-Krise hinaus konsequent weitergehen. Anders als die Wissenschaftlichen Dienste des Deutschen Bundestages (2020, 4-5) meinen, ist dafür auch keine Änderung des Grundgesetzes, sondern „nur“ eine Anpassung der Geschäftsordnung notwendig. So hat der Deutsche Bundestag eine weitgehende „Virtualisierung“ der Arbeit von Bundestagsauschüssen in der CoronaKrise allein durch die Änderung seiner Geschäftsordnung ermöglicht: An Ausschusssitzungen und -beratungen können Abgeordnete über elektronische Kommunikationsmittel teilnehmen ( $§ 126 a$ Absatz 2 Geschäftsordnung des Deutschen Bundestages). Darüber hinaus kann mittels elektronischer Kommunikationsmedien eine Beschlussfassung in Ausschüssen erfolgen ( $\S 126 \mathrm{a}$ Absatz 3 GOBT) und die Öffentlichkeit von Ausschusssitzungen hergestellt werden (§ 126a Absatz 4 Geschäftsordnung des Deutschen Bundestages). Hieran lässt sich auch für die technische und rechtliche Ausgestaltung eines virtuellen Plenums anknüpfen.

\section{Fazit}

Die Rechts- und Verfassungsordnung reguliert das Recht und seine Medien, indem sie entweder normativ lernt oder an einem bestimmten Medienstand normativ festhält. Dabei werden die rechtlichen Möglichkeiten und Grenzen der Verwendung von Augmented und Virtual Reality teilweise auch schlicht durch die normative Kraft des Faktischen bestimmt, die sich in der Kommunikationstechnik- und Medienentwicklung besonders dynamisch entfaltet. Auf diese medialen Dynamiken kann und muss die Rechtsordnung gerade auch im politischen Verfassungsprozess durch die Realitätsverschiebung von Rechtsfiguren und Rechtsinstituten reagieren. Diesen medialen Lernprozess kann die Rechts- und Verfassungsordnung vollkommen problemlos gestalten, weil Rechtsfiguren und Rechtsinstitute selbst einen normativ-konstruktiven Charakter aufweisen, der immer auch durch eine bestimmte Medienverfassung geprägt wird. Deshalb steht die Verfassungs- 
und Rechtsordnung neuen Medienentwicklungen grundsätzlich offen gegenüber, selbst wenn sie im Einzelfall an einer überkommenen Medienkonstellation festhalten muss, um die Funktionsfähigkeit von Rechtsfiguren und Rechtsinstituten zu gewährleisten. Mit Blick auf die verfassungsrechtliche Dimension von Augmented und Virtual Reality ist am Beispiel der Versammlungsfreiheit zunächst deutlich geworden, dass sich die Verfassungsordnung im Interesse einer möglichst effektiven Grund- und Freiheitsentwicklung auf sehr weitgehende rechtliche Realitätsverschiebungen einlässt. Demgegenüber gestaltet sich der mediale Lernprozess im Staatsorganisationsrecht sehr viel komplexer und differenzierter: Im Rahmen der Exekutive bestehen breite Möglichkeiten für den Einsatz und die Verwendung von Virtual Reality. Im Rahmen der Judikative und der Legislative deuten sich jedenfalls erste Möglichkeiten für mediale Realitätsverschiebungen durch Virtual Reality an.

\section{Literatur}

Andreas, Michael, Dawid Kasprowicz und Stefan Rieger. „Unterwachen und Schlafen: Einleitung“. Unterwachen und Schlafen: Anthropophile Medien nach dem Interface. Hg. Michael Andreas, Dawid Kasprowicz und Stefan Rieger. Lüneburg: meson press, 2018. 7-31.

Baecker, Dirk. Studien zur nächsten Gesellschaft. Frankfurt a.M.: Suhrkamp, 2007.

Baecker, Dirk. 4.0 oder Die Lücke die der Rechner lässt. Berlin: Merve, 2018.

Bailenson, Jeremy. Experience On Demand. What Virtual Reality is, How It Works, And What It Can Do. New York, London: W. W. Norton, 2018.

Benjamin, Walter. Das Kunstwerk im Zeitalter seiner technischen Reproduzierbarkeit. 5. Auflage. Berlin: Suhrkamp, 2017 [1936].

BGH (Bundesgerichtshof). „Urteil vom 24. Februar 2015 - VI ZR 106/13“. Neue Juristische Wochenschrift 68.22 (2015): 1601-1603.

Blühdorn, Ingolfur. Simulative Demokratie. Neue Politik nach der postdemokratischen Wende. Berlin: Suhrkamp, 2013.

Buchsteiner, Jochen. „Deutsches Vorbild“. Frankfurter Allgemeine Zeitung, Nr. 95. (23. April 2020): 3.

BVerfG (Bundesverfassungsgericht). „Beschluss vom 15. Dezember 1983 - 1 BvR 209, 269, 362, 420, 440, 484/83“. Entscheidungen des Bundesverfassungsgerichts. Hg. Mitglieder des Bundesverfassungsgerichts. Band 65. Tübingen: Mohr Siebeck, 1984. 1-71.

BVerfG (Bundesverfassungsgericht). „Beschluss vom 14. Mai 1985 - 1 BvR 233, 341/81“. Entscheidungen des Bundesverfassungsgerichts. Hg. Mitglieder des Bundesverfassungsgerichts. Band 69. Tübingen: Mohr Siebeck, 1985. 315-372.

BVerfG (Bundesverfassungsgericht). „Beschluss vom 24. Oktober 2001 - 1 BvR 1190/90, 2173/ 93, 433/96“. Entscheidungen des Bundesverfassungsgerichts. Hg. Mitglieder des Bundesverfassungsgerichts. Band 104. Tübingen: Mohr Siebeck, 2002. 92-126. 
BVerfG (Bundesverfassungsgericht). „Urteil vom 27. Februar 2008 - 1 BvR 370, 595/07“.

Entscheidungen des Bundesverfassungsgerichts. Hg. Mitglieder des

Bundesverfassungsgerichts. Band 120. Tübingen: Mohr Siebeck, 2008. 274-350.

BVerfG (Bundesverfassungsgericht). „Urteil vom 3. März 2009 - 2 BvC 3, 4/07“.

Entscheidungen des Bundesverfassungsgerichts. Hg. Mitglieder des

Bundesverfassungsgerichts. Band 123. Tübingen: Mohr Siebeck, 2010. 39-88.

BVerfG (Bundesverfassungsgericht). „Urteil vom 22. Februar 2011 - 1 BvR 699/06“.

Entscheidungen des Bundesverfassungsgerichts. Hg. Mitglieder des

Bundesverfassungsgerichts. Band 128. Tübingen: Mohr Siebeck, 2011. 226-278.

BVerfG (Bundesverfassungsgericht). „Beschluss vom 18. Juli 2015 - 1 BvQ 25/15“. Neue

Juristische Wochenschrift 68.34 (2015): 2485-2486.

Deutscher Bundestag-Wissenschaftliche Dienste. Virtuelles Parlament. Verfassungsrechtliche

Bewertung und mögliche Grundgesetzänderung. WD 3-3000-084/20. Berlin: Deutscher

Bundestag, 31. März 2020.

Feigenson, Neal. „Too Real? The Future of Virtual Reality Evidence“. Law \& Policy 28.2 (2006):

271-293.

Foucault, Michel. Überwachen und Strafen. Die Geburt des Gefängnisses. Frankfurt a.M.:

Suhrkamp, 1976.

Giesen, Richard. „Robotik und Arbeitsrecht“. Rechtswissenschaft 9.4 (2018), 431-452.

Hilgert, Felix, und Philipp Sümmermann. „Hausverbot für Pokémons? Abwehr virtueller

Gegenstände auf Privatgrundstücken. Eine erste Analyse der möglichen

Anspruchsgrundlagen“. Computer und Recht 32.9 (2016): 580-586.

Ingold, Albert. „,Polizei 2.0‘: Grenzen der behördlichen Öffentlichkeitsarbeit in sozialen

Netzwerken“. Verwaltungsarchiv 108.2 (2017a): 240-265.

Ingold, Albert. „Digitalisierung demokratischer Öffentlichkeiten“. Der Staat 56.4 (2017b):

491-533.

Jacobs, Steffen, Benjamin Lotz und Frank Maschmann. „No-Go für Pokémon Go? -

Abwehransprüche von Betriebsinhabern gegen Betreiber von augmented-reality-Software“.

BetriebsBerater 71.49 (2016): 2997-2999.

Jellinek, Georg. Allgemeine Staatslehre. 3. Auflage. Berlin: Häring, 1914.

Joseph, Gregory P. „Virtual Reality Evidence“. Boston University Journal of Sciences \&

Technology Law 212 (1996): 1-28.

Kaube, Jürgen. „Ist das Kunst oder muss das weg?“. Frankfurter Allgemeine Zeitung, Nr. 28

(2. Februar 2018): 9.

Kelly, Kevin. The Inevitable. Understanding the 12 Technological Forces That will Shape Our Future. New York: Penguin, 2016.

Kersten, Jens. „IT und Demokratie“. Innovationen im Recht. Hg. Wolfgang Hoffmann-Riem. Baden-Baden: Nomos, 2016. 305-336.

Kersten, Jens. Schwarmdemokratie. Der digitale Wandel des liberalen Verfassungsstaats. Tübingen: Mohr Siebeck, 2017a.

Kersten, Jens. „Relative Rechtssubjektivität. Über autonome Automaten und emergente Schwärme“. Zeitschrift für Rechtssoziologie 37.1 (2017b): 8-25.

Kersten, Jens. „Anonymität in der liberalen Demokratie“. Juristische Schulung 57.3 (2017c): 193-203.

Kersten, Jens. „KI-Kunst - Künstliche Intelligenz und künstlerische Freiheit“. Territorialität und Personalität. Festschrift für Moris Lehner zum 70. Geburtstag. Hg. Roland Ismer, 
Ekkehart Reimer, Alexander Rust und Christian Waldhoff. Köln: Otto Schmidt, 2019. 437-452.

Kingreen, Thorsten und Ralf Poscher. Grundrechte. Staatsrecht II. 34. Auflage. Heidelberg:

C. F. Müller, 2018.

Kohle, Hubertus. Museen digital. Eine Gedächtnisinstitution sucht den Anschluss an die Zukunft. Heidelberg: Heidelberg University Publishing, 2018.

Lanier, Jaron. Anbruch einer neuen Zeit. Wie Virtual Reality unser Leben und unsere Gesellschaft verändert. Hamburg: Hoffmann und Campe, 2018.

Latour, Bruno. Das Parlament der Dinge. Für eine politische Ökologie. Frankfurt a.M.: Suhrkamp, 2010.

Lenzen, Manuela. Künstliche Intelligenz. Was sie kann \& was uns erwartet.

2. Auflage. München: C. H. Beck, 2018.

Luhmann, Niklas. Rechtssoziologie. 3. Auflage. Opladen: Westdeutscher Verlag, 1987.

Manow, Philip. Im Schatten des Königs. Die politische Anatomie demokratischer Repräsentation. 2. Auflage. Frankfurt a.M.: Suhrkamp, 2015.

Martini, Mario. „Transformation der Verwaltung durch Digitalisierung“. Die Öffentliche Verwaltung 70.11 (2017a): 444-455.

Martini, Mario. „Algorithmen als Herausforderung für die Rechtsordnung“. JuristenZeitung 72.21 (2017b): 1017-1025.

Martini, Mario, und David Nink. „Wenn Maschinen entscheiden . . . - vollautomatisierte Verwaltungsverfahren und der Persönlichkeitsschutz“. Neue Zeitschrift für Verwaltungsrecht - Extra 36.10 (2017): 1-14.

Marx, Karl, und Friedrich Engels. Manifest der Kommunistischen Partei. 50. Auflage. Berlin: Dietz, 1984 [1848].

Metz, Markus und Georg Seeßlen. Schnittstelle Körper. Berlin: Matthes \& Seitz, 2018.

Möllers, Christoph. Demokratie - Zumutungen und Versprechen. Berlin: Wagenbach 2008.

Möllers, Christoph. „Drei Dogmen der etatistischen Demokratietheorie“. Postnationale Demokratie, Postdemokratie, Neoetatismus. Wandel klassischer Demokratievorstellungen in der Rechtswissenschaft. Hg. Hans Michael Heinig und Jörg Philipp Terhechte. Tübingen: Mohr Siebeck, 2013.131-135.

Münkler, Laura. „Metaphern im Recht. Zur Bedeutung organischer Vorstellungen von Staat und Recht“. Der Staat 55.2 (2016): 181-211.

OLG (Oberlandesgericht) Hamm. „Urteil vom 9. Dezember 2004 - 21 U 58/04“. BeckRechtsprechung 2004, 30347437.

OLG (Oberlandesgericht) Jena. „Urteil vom 30. November 2011 - 7 U 178/10“. BeckRechtsprechung 2012, 4774.

OLG (Oberlandesgericht) München. „Urteil vom 13. Mai 2016 - 10 U 4529/15“. BeckRechtsprechung 2016, 10166.

Plaum, Wätzold. Die Wiki-Revolution. Absturz und Neustart der westlichen Demokratie. Berlin: Rotbuch, 2013.

Prütting, Jens, und Maren Wilke. „Pokémon GO - Ein rechtlich bedenkliches Vergnügen. Datenschutz- und haftungsrechtliche Analyse“. Kommunikation und Recht 19.9 (2016): 545-550.

Ramge, Thomas. Mensch und Maschine. Wie künstliche Intelligenz und Roboter unser Leben verändern. 4. Auflage. Stuttgart: Reclam, 2018.

Rauterberg, Hanno. Wie frei ist die Kunst? Der neue Kulturkampf und die Krise des Liberalismus. Berlin: Suhrkamp, 2018. 
Rieger, Stefan. Die Enden des Körpers. Versuch einer negativen Prothetik. Wiesbaden: Springer, 2019.

Roßmann, Robert, und Georg Mascolo. „Ein Rettungspaket für den Bundestag“. Süddeutsche Zeitung (3. April 2020). https://www.sueddeutsche.de/politik/ corona-bundestag-schaeuble-1.4867460 (7. April 2020).

Scheiner, Bruce. Click Here to Kill Everybody. Security and Survival in a Hyper-connected World. New York: W. W. Norton, 2018.

Schönberger, Christoph. „Vom Verschwinden der Anwesenheit in der Demokratie. Präsenz als bedrohtes Fundament von Wahlrecht, Parteienrecht und Parlamentsrecht“. JuristenZeitung 71.10 (2016): 486-494.

Schönberger, Christoph, und Sophie Schönberger. „Regiert bald ein Notausschuss?“. Frankfurter Allgemeine Zeitung, Nr. 73 (26. März 2020): 9.

Siehr, Angelika. Das Recht am öffentlichen Raum. Theorie des öffentlichen Raumes und die räumliche Dimension der Freiheit. Tübingen: Mohr Siebeck, 2016.

Söbbing Thomas. „Pokémon Go und seine rechtlichen Implikationen. Was tun gegen störende Pokéstops?“. MultiMedia und Recht 19.11 (2016): 719-723.

Solmecke, Christian, und Sibel Kocatepe. „Google Glass - Der Gläserne Mensch 2.0. Die neueste technische Errungenschaft - ein Fluch oder eine Herausforderung?“. Zeitschrift für Datenschutz 4.1 (2014): 22-27.

Tinnefeld, Robert. „Zur Verantwortung des Spieleanbieters für reale Störungen durch virtuelles Spiel - oder: Wie wird man die Pokémon wieder los?“. Kommunikation und Recht 19.9 (2016): 551-556.

Vismann, Cornelia. Medien der Rechtsprechung. Frankfurt a.M.: Fischer, 2011.

Vismann, Cornelia. Das Recht und seine Mittel. Ausgewählte Schriften. Frankfurt a.M., 2012.

Volland, Holger. Die kreative Macht der Maschinen. Warum Künstliche Intelligenzen bestimmen, was wir morgen fühlen und denken. Weinheim, Basel: Beltz, 2018. 